\title{
Estudio Transversal Comparativo de la Relación Maxilo- Mandibular de McNamara Aplicadas a Sujetos Mexicanos
}

\author{
Transversal Comparative Study of McNamara Maxillofacial \\ Mandibular Ratio Applied in Mexican Subjects
}

\begin{abstract}
Jairo Mariel Cárdenas*; José Manuel Guijarro Bañuelos*; Wulfrano Sánchez Meraz*; Humberto Mariel Murga*; Gylmar Mariel Cárdenas*; María Esther Navarro Rincón-Gallardo* \& Francisco Javier Gutiérrez Cantú*
\end{abstract}

MARIEL, C. J.; GUIJARRO, B. J. M.; SÁNCHEZ, M. W.; MARIEL, M. H.; MARIEL, C. G.; NAVARRO, R. M. E. \& GUTIÉRREZ, C. F. J. Estudio transversal comparativo de la relación maxilo-mandibular de McNamara aplicadas a sujetos mexicanos. Int. J. Morphol., 34(2):454-459, 2016.

RESUMEN: Las características antropológicas entre diversas razas en las cuales se incluyen etnia, sexo y edad en el mundo varían, es preciso analizar que un estudio establecido para una población no puede ser usado para otra, ya que presenta formas y características diferentes; es por esto que existe la necesidad de realizar estudios en diversas razas comparándolos con las normas establecidas. El objetivo fue comparar la relación maxilo-mandibular de McNamara aplicadas en sujetos mexicanos. Se analizaron 60 radiografías laterales de cráneo con trazados de McNamara. Se identificaron diferencias en los patrones genéticos de crecimiento de los caucásicos y los mexicanos. Nuestros resultados muestran un crecimiento típico de los pacientes clase II esqueletal con maxilar hipotónico posteroanteriormente, una mandíbula disminuida y poca altura vertical, esto como consecuencia de una falta de crecimiento del tercio medio facial por una pobre ventilación aérea.

PALABRAS CLAVE: McNamara; Relación Maxilo-mandibular; Cefalometría.

\section{INTRODUCCIÓN}

Las características antropológicas entre diversas razas en las cuales se incluyen etnia, sexo y edad en el mundo varían, desde la morfología del cuerpo hasta la morfología craneana; es preciso analizar que un estudio establecido para una población no puede ser usado para otra, ya que presenta formas y características diferentes (McNamara \& Ellis, 1988; Athanasiou, 1997; Rios Sánchez, 2007).

La cefalometría es un método auxiliar de diagnóstico en ortodoncia con el cual se obtienen planos de referencia empleando puntos craneo-cefalométricos, en la mayoría de los casos dichos planos se relacionan obteniendo medidas lineales y angulares (Broadbent, 1931; Quirós, 1998; Companioni Bachá et al., 2008; Gómez Gómez et al., 2011).

Los primeros estudios métricos de la cabeza fueron realizados por Leonardo Da Vinci, en 1452, quien estableció proporciones entre líneas y segmentos (Rios Sánchez).
Las investigaciones realizadas con fines antropológicos con el objeto de determinar características étnicas, sexo, edad, etc; tienen como punto de partida los trabajos de Camper, que en 1780 describió por primera vez la utilidad del ángulo formado por la intersección de un plano trazado desde la base de la nariz al conducto auditivo externo (plano de Camper) con el plano tangente al perfil facial (Behbehani et al., 2006).

La cefalometría radiológica surgió en 1934 por Hofrath en Alemania y Broadbent en Estados Unidos. Ésta significó la posibilidad de utilizar una nueva técnica en el estudio de la maloclusión y las discrepancias esqueléticas (Behbehani et al.). En un principio, la cefalometría tenía como objetivo el estudio de los patrones de crecimiento craneofacial, posteriormente se comprobó que la cefalometría podía emplearse para valorar las proporciones dentofaciales y descifrar las bases anatómicas de la maloclusión (Barahona \& Benavides, 2006). 
El principio del análisis cefalométrico consiste en comparar al paciente con un grupo de referencia normal para poder detectar cualquier diferencia entre las relaciones dentofaciales del paciente y las que cabría esperar en su grupo étnico o racial. El objetivo del análisis cefalométrico es el estudio de las relaciones horizontales y verticales de los cinco componentes funcionales más importantes de la cara: el cráneo y la base craneal, el maxilar, la dentición, los procesos alveolares y la mandíbula (Barahona \& Benavides). La necesidad creciente de realizar un diagnóstico adecuado de las condiciones óseas del paciente y su relación con los tejidos blandos y dentarios unidos a la aparición de la tecnología necesaria para obtención de los rayos X, se han convertido en el impulso sistemático que propició el vertiginoso desarrollo de este sistema de diagnóstico (Companioni Bachá et al.).

El análisis de W.B. Downs fue desarrollado en la Universidad Illinois en el año de 1948. Se basó en las proporciones faciales y esqueléticas de un grupo de referencia de 20 adolescentes blancos no sometidos a tratamiento y seleccionados por tener una oclusión dental ideal. Se considera que este es el primer análisis usado en el diagnóstico ortodóntico. Este estudio no valora anomalías de volumen como sí ocurre en otros análisis como el de Steiner. Para su análisis, Downs utiliza el plano de Frankfort. A partir de los perfiles faciales determina que la posición de la mandíbula es la que determina si las caras son o no armónicas. Dando a conocer 4 grupos faciales: retrognático, mesognático, prognático y prognatismo verdadero (Barahona \& Benavides; Downs, 1948, 1952).

Cecil C. Steiner en los años 50 se basó en los trabajos de Northwest, Downs, Wylie Reídle, Margolis y otros. Ofreció pautas específicas para poder aplicar las mediciones cefalométricas a la planificación por medio del establecimiento de metas de tratamiento específico (Barahona \& Benavides; Steiner, 1955). El análisis de Ricketts es un análisis global de 11 factores en el que se emplean mediciones específicas para localizar el mentón en el espacio; localizar el maxilar a través de la convexidad de la cara, localizar la relación dental en la cara y estudiar el perfil facial. En el análisis de Ricketts, las principales líneas de referencia son la horizontal de Frankfort, la línea nasionbasion y la vertical pterigoidea. Este método no se limita a analizar la situación actual del paciente, sino que permite predecir los efectos del crecimiento futuro y el tratamiento (Barahona \& Benavides; Ricketts, 1957, 1981).

McNamara publica en 1984 su estudio, el cual utiliza 3 recursos: Estándares de Bolton (Broadbent, 1937), Muestra de Burlington y Muestra de Ann Arbor. Este estudio consiste en un conjunto de mediciones lineales y an- gulares, útiles para el diagnóstico y planificación del tratamiento de un paciente, y así mismo comparar las medidas con normas ya establecidas (McNamara, 1984; Rios Sánchez). Dicho análisis es introducido por la necesidad de crear un método de análisis cefalométrico que no solo sea más específico en la posición de los dientes con el hueso alveolar, sino además en la relación del maxilar inferior con la base del cráneo (Rios Sánchez). McNamara basa su estudio en doce puntos cefalométricos, los cuales son los que se describen en la Figura 1.

Se han descrito investigaciones donde se utiliza el estudio de McNamara para comparar la raza caucásica con otras razas tales como japoneses, mestizos, asiáticos, turcos, entre otros, pero nunca se ha realizado con mexicanos, es por esto que surge la idea de ésta investigación, donde se evaluaron las medidas maxilomandibulares en sujetos mexicanos y las medidas obtenidas en el estudio de McNamara comparando si existen variaciones entre ambas (Broadbent, 1931, 1937; Rios Sánchez; Cooke \& Wei, 1989; Romero, 2004; Gu et al., 2011; Uysal et al., 2012).

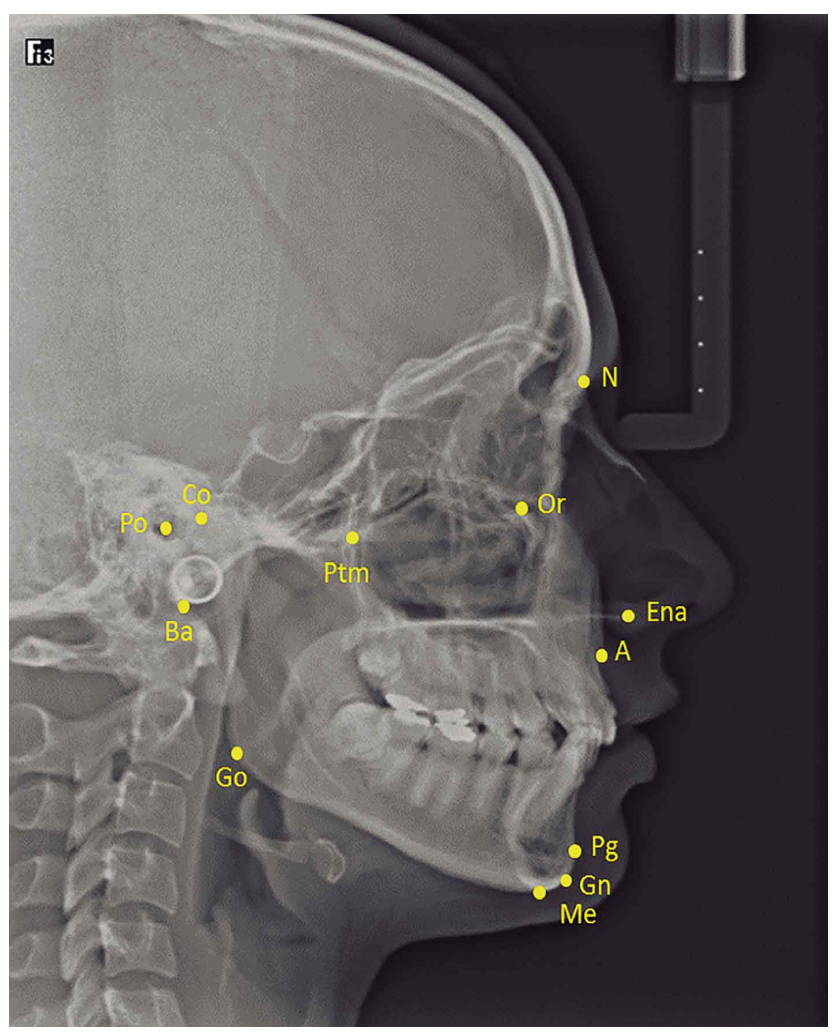

Fig. 1. Puntos cefalométricos para el estudio de McNamara. $\mathrm{N}=$ Nasion, $\mathrm{Or}=$ Orbital, $\mathrm{Co}=$ Condilion, $\mathrm{Po}=\mathrm{Porion}, \mathrm{Ptm}=$ Fisura Pterigomaxilar, $\mathrm{Ba}=\mathrm{B}$ asion, $\mathrm{Go}=$ Gonion, Ena $=$ Espina Nasal Anterior, $\mathrm{A}=$ Punto $\mathrm{A}, \mathrm{Pg}=$ Pogonion, $\mathrm{Gn}=$ Gnation, $\mathrm{Me}=$ Menton. 


\section{MATERIAL Y MÉTODO}

Se analizaron 60 radiografías laterales de cráneo obtenidas del banco de radiografías del Posgrado de Ortodoncia y Ortopedia Dentomaxilofacial de la UASLP (Universidad Autónoma de San Luis Potosí). Se estudiaron dos grupos (30 hombres y 30 mujeres). A cada radiografía se le trazó el estudio cefalométrico de McNamara. Se compararon los valores obtenidos con la norma establecida y se realizó un análisis estadístico de los resultados.

\section{RESULTADOS}

Las mujeres mexicanas en relación con las caucásicas presentan un maxilar más prominente con respecto a la base de cráneo, mientras que los hombres presentan un maxilar más pequeño o retruido en relación a la base del cráneo, por lo tanto un perfil más convexo comparado con los hombres caucásicos (Tabla I).

Comparación de medidas cefalométricas. Co-Gn: La longitud total de la mandíbula en mujeres y hombres caucásicos es mayor en comparación de los mexicanos (Tabla I).
Co-A: Se observó que tanto en hombres como en mujeres mexicanas hay un desarrollo menor en sentido anteroposterior del maxilar con respecto al cóndilo mandibular; dándo como resultado un maxilar hipoplasico en comparación con los caucásicos. Esta medida en conjunto con la medida de Nasion perpendicular al punto A, ayuda a ubicar anteroposteriormente al maxilar. En relación a los valores obtenidos la diferencia maxilo mandibular esta disminuida en personas mexicanas con respecto a la norma (Tabla II).

Ena-me: esta medida nos indica el crecimiento vertical de la parte anterior del tercio inferior de la cara; se obtuvo que los varones mexicanos presentan esta medida disminuida en relación a los índices de McNamara mientras que las cifras de las mujeres están dentro de la norma (Tabla II).

Po-Or/Go-Me: Éste punto interpreta la inclinación de la mandíbula con respecto a la base del cráneo; se observó un leve aumento de este ángulo en hombres y mujeres mexicanas por lo que nos indica que hay una tendencia de crecimiento vertical mandibular (Tabla II).

Tabla I. Resultados comparativos de la maxila contra la base del cráneo según McNamara en comparación con mexicanos.

\begin{tabular}{lcccccccc}
\hline \multirow{2}{*}{ Maxila contra base del craneo } & \multicolumn{3}{c}{ Caucásicos } & \multicolumn{3}{c}{ Mexicanos } \\
\cline { 2 - 9 } & \multicolumn{2}{c}{ Mujeres } & \multicolumn{2}{c}{ Hombres } & \multicolumn{2}{c}{ Mujeres } & \multicolumn{2}{c}{ Hombres } \\
& Media & DE & Media & DE & Media & DE & Media & DE \\
\hline Nasion Perpendicular-Punto A (Nap-A) & 0,4 & $\pm 2,3$ & 1,1 & $\pm 2,7$ & 103,9 & $\pm 2,5$ & 103,5 & $\pm 2,7$ \\
\hline
\end{tabular}

Tabla II. Comparativa de la relación maxilo mandibular según McNamara en comparación con mexicanos.

\begin{tabular}{|c|c|c|c|c|c|c|c|c|}
\hline \multirow{3}{*}{ Relacion maxilo mandibular } & \multicolumn{4}{|c|}{ Caucásicos } & \multicolumn{4}{|c|}{ Mexicanos } \\
\hline & \multicolumn{2}{|c|}{ Mujeres } & \multicolumn{2}{|c|}{ Hombres } & \multicolumn{2}{|c|}{ Mujeres } & \multicolumn{2}{|c|}{ Hombres } \\
\hline & Media & DE & Media & DE & Media & DE & Media & DE \\
\hline Longitud mandibular efectiva (Co-Gn) & 120,2 & $\pm 5,3$ & 132,3 & $\pm 6,8$ & 103,9 & $\pm 5,4$ & 103,5 & $\pm 19,9$ \\
\hline Longitud maxilar efectiva (Co-A) & 91 & $\pm 4,3$ & 99,8 & \pm 6 & 78,3 & $\pm 4,1$ & 81,5 & $\pm 7,9$ \\
\hline Diferencia maxilo-mandibular (Co-A/Co-Gn) & 29,2 & $\pm 3,3$ & 32,5 & \pm 4 & 25,63 & $\pm 4,3$ & 25,3 & $\pm 6,4$ \\
\hline Altura facia 1 anteroinferior (Ena-Me) & 66,7 & $\pm 4,1$ & 74,6 & \pm 5 & 61,7 & $\pm 5,3$ & 44,5 & $\pm 6,6$ \\
\hline Ángulo del plano mandibular (Po-Or/ Go-Me) & 22,7 & $\pm 4,3$ & 21,3 & $\pm 3,9$ & 26,4 & $\pm 6,1$ & 25,4 & $\pm 4,1$ \\
\hline Ángulo del eje facial (Ba-N/ Pt-GnI) & 0,2 & $\pm 3,2$ & 0,5 & $\pm 3,5$ & 0,8 & $\pm 4,0$ & 0,5 & $\pm 3,4$ \\
\hline
\end{tabular}

Tabla III. Resultados del estudio de McNamara en pacientes mexicanos según la mandíbula contra la base del cráneo.

\begin{tabular}{lcccccccc}
\hline & \multicolumn{4}{c}{ Caucásicos } & \multicolumn{3}{c}{ Mexicanos } \\
\cline { 2 - 8 } Mandíbula contra base del craneo & \multicolumn{2}{c}{ Mujeres } & \multicolumn{2}{c}{ Hombres } & \multicolumn{2}{c}{ Mujeres } & \multicolumn{2}{c}{ Hombres } \\
& Media & DE & Media & DE & Media & DE & Media & DE \\
\hline Nasion Perpendicular a Pg (Nap-Pg) & $-1,8$ & $\pm 4,5$ & $-0,3$ & $\pm 3,8$ & $-0,7$ & $\pm 5,5$ & $-1,8$ & $\pm 4,6$ \\
\hline
\end{tabular}


Ba-N/Pt-Gn: Éste ángulo nos da como referencia donde se ubica el mentón en relación a la base del cráneo, de acuerdo a los resultados obtenidos se pudo observar que la mujer mexicana tiende a tener un mentón retrognático con respecto a las caucásicas (Tabla II).

Nap-Pg: El análisis de McNamara indica que es la posición anteroposterior del maxilar con la base del cráneo, en el presente estudio se observó que en las mexicanas existe un menor crecimiento anteroposterior del maxilar, por lo tanto se infiere que hay; por otro lado los hombres mexicanos están en la norma de acuerdo al estudio (Tabla III).

Incisivo Sup-vertical al punto A: Esta medida nos indica la posición anteroposterior del incisivo superior con respecto al maxilar; como resultado nos indica que tanto en mujeres como en hombres mexicanos no hubo un cambio apreciable con respecto a los caucásicos (Tabla IV).

Incisivo Inf-plano A-Pg: Esta medida refleja la distancia del incisivo inferior con respecto al perfil óseo, y podemos observar que en los resultados, los mexicanos poseen un incisivo inferior ligeramente más protruído que los caucásicos (Tabla IV).
Faringe superior: Se encontró que en las personas caucásicas tanto mujeres como hombres poseen un tubo aéreo superior de mayor diámetro a comparación de los mexicanos (Tabla V).

Faringe posterior: En el análisis realizado por McNamara esta medida indica el diámetro de la vía aérea inferior, y de acuerdo a los resultados que se observaron, las mujeres y hombres mexicanos tienen una vía aérea inferior más reducida a comparación de los caucásicos; también podemos observar, que los hombres mexicanos poseen una vía aérea inferior más permeable a comparación de las mujeres mexicanas (Tabla V).

La Tabla IV, indica cómo se encuentra colocado el incisivo superior con respecto al maxilar y el incisivo inferior con la mandíbula, en ambos casos se obtuvo como resultado que tanto en hombres como mujeres mexicanas estos se encuentran más protruidos que en los caucásicos.

En los puntos anteriores (Tabla V) que indican el diámetro de la vía aérea, se observó que la faringe superior e inferior en mexicanos se encuentra disminuida, por lo tanto hay menor ventilación que en los caucásicos, en consecuencia se presenta una deficiencia en el crecimiento del tercio medio e inferior por la falta de función.

Tabla IV. Comparativa de la dentición en pacientes mexicanos según McNamara.

\begin{tabular}{lcccccccc}
\hline & \multicolumn{3}{c}{ Caucásicos } & \multicolumn{3}{c}{ Mexicanos } \\
\hline Denticion & \multicolumn{2}{c}{ Mujeres } & \multicolumn{2}{c}{ Hombres } & \multicolumn{2}{c}{ Mujeres } & \multicolumn{2}{c}{ Hombres } \\
& Media & DE & Media & DE & Media & DE & Media & DE \\
\hline Incisivo sup,-Vertical del punto A & 5,4 & $\pm 1,7$ & 5,3 & \pm 2 & 5,8 & $\pm 2,1$ & 5,5 & $\pm 2,4$ \\
Incisivo inf,-Plano A-Po & 2,7 & $\pm 1,7$ & 2,3 & $\pm 2,1$ & 3,4 & $\pm 2,1$ & 3 & $\pm 1,7$ \\
\hline
\end{tabular}

Tabla V. Resultados comparativos entre pacientes mexicanos y caucásicos según la norma de McNamara.

\begin{tabular}{lcccccccc}
\hline \multirow{2}{*}{ Vías aéreas } & \multicolumn{4}{c}{ Caucásicos } & \multicolumn{4}{c}{ Mexicanos } \\
\cline { 2 - 9 } & \multicolumn{2}{c}{ Mujeres } & \multicolumn{2}{c}{ Hombres } & \multicolumn{2}{c}{ Mujeres } & \multicolumn{2}{c}{ Hombres } \\
& Media & DE & Media & DE & Media & DE & Media & DE \\
\hline Faringe superior & 17,4 & $\pm 3,4$ & 17,4 & $\pm 4,3$ & 11,5 & $\pm 2,2$ & 10,9 & $\pm 3,2$ \\
Faringe posterior & 11,3 & $\pm 3,3$ & 13,5 & $\pm 4,3$ & 10,1 & $\pm 2,2$ & 11,1 & $\pm 2,8$ \\
\hline
\end{tabular}

\section{DISCUSIÓN}

En el campo de la investigación se han desarrollado diversos estudios cefalométricos, esqueletales, faciales y dentales entre determinados grupos raciales y étnicos, con estos estudios demostramos al final un objetivo para realizar un tratamiento ortodóntico o bien una alternativa, pero lo importante es a tratar a los pacientes de acuerdo a sus carac- terísticas individuales para lograr así el resultado adecuado de cualquier tratamiento.

La presente investigación compara una muestra de personas mexicanas con otra muestra desarrollada en caucásicos (cefalometría de McNamara) para buscar algu- 
nas relaciones cefalométricas acorde a nuestra población. Se puede afirmar con el presente estudio que si hay una diferencia notable en los patrones genéticos de crecimiento de los caucásicos a los mexicanos, por lo tanto el análisis de McNamara viene a auxiliar en el tratamiento ortodóntico de un paciente mexicano pero no hay que regirnos en sus resultados, ya que el paciente mexicano tiende a tener un crecimiento típico de los pacientes clase II esqueletal con maxilar hipotónico posteroanteriormente, una mandíbula también disminuida y poca altura vertical, esto como consecuencia de una falta de crecimiento del tercio medio facial por una pobre ventilación aérea (McNamara; McNamara \& Ellis). Wu et al. (2007) realizaron un estudio comparativo en población china donde se encontraron diferencias significativas de raza tanto en pacientes masculinos como femeninos (Steiner, 1955). Por otro lado en un estudio realizado por Rios Sánchez con personas de raza mestiza no presentaron diferencias estadísticamente significativas en comparación al estudio de McNamara pero si se encontraron diferencias significativas entre la muestra de ambos sexos. Nuestros resultados muestran que en la población mexicana si se observan diferencias en cuanto al sexo pero también en general con la raza caucásica, lo que se infiere que es debido a la mezcla interracial que ha habido a lo largo de la historia en ambas razas.

Horna León (2004) en su estudio realizado en una población de hombres mestizos con dentición permanente encontraron que la muestra estudiada presentaba ambos maxilares más grandes, un crecimiento en sentido horario con hiperdivergencia, una protrusión y extrusión dentaria y una mayor distancia de la orofaringe por posición anterior de la lengua. Nuestra investigación concuerda con Horna León, ya que los resultados nos muestran que las mujeres mexicanas presentan un maxilar más prominente que las caucásicas, pero una mandíbula disminuida, lo mismo pasó con los hombres mexicanos.

En otro estudio realizado por Behbehani et al., en donde se comparan adolescentes kuwaitíes, con adolescentes de raza blanca los resultados muestran que existen diferencias significativas entre la población de Kuwait y de la población caucásica de la mayoría de las variables analizadas, el sexo no produjo ningún efecto significativo en ninguna de las variables estudiadas. La población kuwaití tiene labios más llenos, más convexidad facial, mayor protrusión dental, una mandíbula más retruída y pequeña y una altura facial más corta en comparación de los caucásicos; por el contrario en nuestro estudio el sexo si representa un dato significativo ya que existen resultados muy distintos entre hombres y mujeres mexicanas.

La cefalometría de McNamara (1984) depende más de medidas lineales que angulares, lo cual facilita el estudio ortopédico, analiza la relación intermaxilar no solo en sentido sagital si no que le agrega el estudio de los cambios y alteraciones en sentido vertical, cuantificando la incidencia de los cambios en sentido sagital. Es útil para estudiar superficialmente las vías aéreas y las consecuencias que pudieran tener estas.

A diferencia de otros estudios como Steiner que se basa más en medidas angulares por lo que es más complicada su interpretación. La cefalometría de McNamara es complementaria para evaluar la relación intermaxilar con la dimensión vertical entre ellos, de gran ayuda en los casos que requieren tratamiento quirúrgico y ortopédico. En este estudio cada paciente es individual y los valores esqueléticos deben ir íntimamente ligados a los tejidos blandos. De acuerdo al resultado obtenido podemos afirmar que las personas mexicanas tienen distintas características craneofaciales con respecto a los caucásicos y diferencias estadísticas entre ambos sexos, esto se debe a distintos factores ya sean hábitos de alimentación, el clima, raza, sexo, entre otros (Athanasiou).

\section{CONCLUSIÓN}

Con respecto al resultado obtenido su puede afirmar que la raza, etnia y sexo son un factor determinante para cualquier tratamiento de ortodoncia y no debemos limitarnos a un solo estudio ya que cada paciente es individual y por lo tanto necesita un tratamiento específico.

MARIEL, C. J.; GUIJARRO, B. J. M.; SÁNCHEZ, M. W.; MARIEL, M. H.; MARIEL, C. G.; NAVARRO, R. M. E. \& GUTIÉRREZ, C. F. J. Transversal comparative study of McNamara maxillofacial mandibular ratio applied in Mexican subjects. Int. J. Morphol., 34(2):454-459, 2016.

SUMMARY: The anthropological characteristics between different races in which ethnicity, sex and age vary in the world, and it becomes necessary to analyze a set for one population study that cannot be used for another, with different shapes and characteristics. There is a need for studies in different races for comparison with established standards. The objective of the research was to compare the McNamara maxilla-mandibular relationship applied in Mexicans subjects and analyze 60 lateral radiographs of the skulls with McNamara traces. Genetic differences in growth patterns of Caucasians and Mexicans were identified. Our results show typical growth of skeletal class II patients with hypotonic posterior jaw, and jaw with diminished vertical height caused by a lack of midface growth the result of poor air flow.

KEY WORDS: McNamara; Maxillo-Mandibular relationship; Cephalometry. 


\section{REFERENCIAS BIBLIOGRÁFICAS}

Athanasiou, A. E. Orthodontic Cephalometry. London, MosbyWolfe, 1997.

Barahona, C. J. B. \& Benavides, S. J. Principales análisis cefalométricos utilizados para el diagnóstico ortodóntico. Rev. Cient. Odontol., 2(1), 2006. Disponible en: http:// revista.colegiodentistas.org/index.php/revistaodontologica/ article/view/24/51

Behbehani, F.; Preston, E. P.; Beeman, C.; Kluemper, G. T. \& Rayens, M. K. Racial variations in cephalometric analysis between Whites and Kuwaitis. Angle Orthod., 76(3):406-11, 2006.

Broadbent, B. H. A new x-ray technique and its application to orthodontia. Angle Orthod., 51(2):93-114, 1931.

Broadbent, B. H. Bolton standards and technique in orthodontic practice. Angle Orthod., 7(4):209-33, 1937.

Companioni Bachá, A.; Rodríguez Quiñónez, M.; Días de Villegas Rushkova, V. \& Otaño Lugo, R. Bosquejo histórico de la cefalometría radiográfica. Rev. Cuba. Estomatol., 45(2), 2008. Disponible en: http://scielo.sld.cu/ scielo.php? script=sci_arttext\&pid=S003475072008000200009

Cooke, M. S. \& Wei, S. H. A comparative study of southern Chinese and British Caucasian cephalometric standards. Angle Orthod., 59(2):131-8, 1989.

Downs, W. B. Variations in facial relationships; their significance in treatment and prognosis. Am. J. Orthod., 34(10):812-40, 1948.

Downs, W. B. The role of cephalometrics in orthodontic case analysis and diagnosis. Am. J. Orthod., 38(3):162-82, 1952.

Gómez Gómez, V.; Fernández López, A. \& Pérez Tejada, H. E. Características cefalométricas presentes en la maloclusión clase I en el Departamento de la DEPeI. Rev. Odontol. Mex., 15(1):14-20, 2011.

Gu, Y.; McNamara, J. A. Jr .; Singler, L. M. \& Baccetti, T. Comparison of craniofacial characteristics of typical Chinese and Caucasian young adults. Eur. J. Orthod., 33(2):205-11, 2011.

Horna León, Y. A. Estudio Comparativo del Patrón Cefalométrico de Mcnamara en una Población de Varones Mestizos en Dentición Permanente. Lima, Universidad Nacional Mayor de San Marcos, 2004.

McNamara, J. A. Jr. A method of cephalometric evaluation. Am. J. Orthod., 86(6):449-69, 1984.
McNamara, J. A. Jr. \& Ellis, E. 3rd. Cephalometric analysis of untreated adults with ideal facial and occlusal relationships. Int. J. Adult Orthodon. Orthognath. Surg., 3(4):221-31, 1988.

Quirós, A O. Análisis cefalométrico para la medición de cambios dentofaciales de Quirós. Acta Odontol. Venez., 36(1), 1998. Disponible en: http://www.actaodontologica.com/ediciones/ 1998/1/cefalometrico_medicion_dentofaciales.asp

Ricketts, R. M. Planning treatment on the basis of the facial pattern and an estimate of its growth. Angle Orthod., 27(1):14-37, 1957.

Ricketts, R. M. Perspectives in the clinical application of cephalometrics. The first fifty years. Angle Orthod., 51(2):11550,1981

Rios Sánchez, C. J. Normas cefalométricas del análisis de McNamara: estudio comparativo entre una población caucásica y una población mestiza. Kiru, 4(2):45-51, 2007.

Romero, G. N. J. Precisión en la Localización de los Puntos Cefalométricos en un Análisis de Radiografía Lateral. Lima, Facultad de Odontología. Universidad Nacional Mayor de San Marcos, 2004.

Steiner, C. S. Cephalometrics for you and me. Am. J. Orthod., 39(10):729-55, 1955.

Uysal, T.; Baysal, A.; Yagci, A.; Sigler, L. M. \& McNamara, J. A. Jr. Ethnic differences in the soft tissue profiles of Turkish and European-American young adults with normal occlusions and well-balanced faces. Eur. J. Orthod., 34(3):296-301, 2012.

Wu, J.; Hägg, U. \& Rabie, A. B. Chinese norms of McNamara's cephalometric analysis. Angle Orthod., 77(1):12-20, 2007.

Dirección para Correspondencia:

Francisco Javier Gutiérrez Cantú

Dirección: Av. Manuel Nava 2

Código Postal: 78290.

San Luis Potosí, S.L.P.

MÉXICO

Email: pacogtz@uaslp.mx

Recibido : 13-07-2015

Aceptado: 25-02-2016 